\title{
Stability of supercooled binary liquid mixtures
}

Toxværd, Søren; Pedersen, Ulf Rørbæk; Schrøder, Thomas; Dyre, J. C.

Published in:

Journal of Chemical Physics

DOI:

10.1063/1.3144049

Publication date:

2009

Document Version

Publisher's PDF, also known as Version of record

Citation for published version (APA):

Toxværd, S., Pedersen, U. R., Schrøder, T., \& Dyre, J. C. (2009). Stability of supercooled binary liquid mixtures. Journal of Chemical Physics, 130(22), 224501. https://doi.org/10.1063/1.3144049

\section{General rights}

Copyright and moral rights for the publications made accessible in the public portal are retained by the authors and/or other copyright owners and it is a condition of accessing publications that users recognise and abide by the legal requirements associated with these rights.

- Users may download and print one copy of any publication from the public portal for the purpose of private study or research.

- You may not further distribute the material or use it for any profit-making activity or commercial gain.

- You may freely distribute the URL identifying the publication in the public portal.

Take down policy

If you believe that this document breaches copyright please contact rucforsk@kb.dk providing details, and we will remove access to the work immediately and investigate your claim. 


\title{
Stability of supercooled binary liquid mixtures
}

\author{
Søren Toxvaerd, ${ }^{\text {a) }}$ Ulf R. Pedersen, Thomas B. Schrøder, and Jeppe C. Dyre \\ Department of Sciences, DNRF Centre "Glass and Time," IMFUFA, Roskilde University, \\ P.O. Box 260, DK-4000 Roskilde, Denmark
}

(Received 6 March 2009; accepted 7 May 2009; published online 8 June 2009)

\begin{abstract}
Recently, the supercooled Wahnström binary Lennard-Jones mixture was partially crystallized into $\mathrm{MgZn}_{2}$ phase crystals in lengthy molecular dynamics simulations. We present molecular dynamics simulations of a modified Kob-Andersen binary Lennard-Jones mixture that also crystallizes in lengthy simulations here, however, by forming pure fcc crystals of the majority component. The two findings motivate this paper that gives a general thermodynamic and kinetic treatment of the stability of supercooled binary mixtures, emphasizing the importance of negative mixing enthalpy whenever present. The theory is used to estimate the crystallization time in a Kob-Andersen mixture from the crystallization time in a series of related systems. At $T=0.40$ we estimate this time to be $5 \times 10^{7}$ time units $(\approx 0.1 \mathrm{~ms})$. A new binary Lennard-Jones mixture is proposed that is not prone to crystallization and faster to simulate than the two standard binary Lennard-Jones mixtures. This is obtained by removing the like-particle attractions by switching to Weeks-Chandler-Andersen type potentials, while maintaining the unlike-particle attraction. (C) 2009 American Institute of Physics.
\end{abstract}

[DOI: $10.1063 / 1.3144049$ ]

\section{INTRODUCTION}

As computers get faster, simulations of the highly viscous liquid phase preceding glass formation become increasingly realistic. In this context it is nice to have a standard model system, something like the Ising model for critical phenomena. For several years binary Lennard-Jones (BLJ) mixtures have served this purpose. In particular, the Wahnström (Wa) and Kob-Andersen (KA) systems ${ }^{1,2}$ because they are easy to simulate and were never found to crystallize. The KA BLJ consists of two types of LennardJones (LJ) particles, $80 \%$ large $(A)$ particles and $20 \%$ small $(B)$ particles. The KA potentials are modifications of the potentials devised by Weber and Stillinger, ${ }^{3}$ who constructed the pair potentials for the binary mixture based of physicalchemical data for the $\mathrm{Ni}_{80} \mathrm{P}_{20}$ alloy. The KA potentials describe a strongly nonideal mixture due to an $A B$ attraction that is three times stronger than the $B B$ attraction. This ensures a large negative mixing enthalpy (and energy), which as detailed below suppresses crystallization into pure $A$ crystals.

Recently, the Wa system was shown to crystallize in lengthy computer runs. ${ }^{4}$ This motivated the present paper that has three purposes. First, we review the general theory of thermodynamic and kinetic stability of supercooled binary mixtures and derive a relation for nucleation times of the solvent in binary mixtures (Sec. II). Second, we detail the crystallization of modified KA (MKA) type systems. Their nucleation times are used to estimate the nucleation time of the KA system to be of the order roughly $0.1 \mathrm{~ms}$ in Argon units (Sec. III). The crystallization of the Wa liquid will be described elsewhere. 5 Finally, we suggest a new KA-type BLJ system that is faster to simulate and which is even less

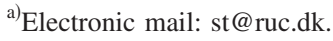

prone to crystallization than the KA system. The idea is to keep the KA system's large negative mixing enthalpy, but remove the $A A$ and $B B$ attractions by adopting WeeksChandler-Andersen (WCA) type potentials between like particles (Sec. IV). Section V gives a brief discussion.

\section{GENERAL TREATMENT}

\section{A. Freezing-point depression}

Consider a binary mixture of $N_{A}$ solvent $(A)$ particles and $N_{B}$ solute $(B)$ particles, with total number of particles $N=N_{A}+N_{B}$. The thermodynamic stability of this system against crystallization is expressed by the melting temperature of a pure $A$ crystal $T_{\text {fus }, A}$ as a function of the concentration of $A$ particles. The latter quantity is conveniently expressed in terms of the fraction $x_{B}=N_{B} / N\left(x_{A}+x_{B}=1\right)$. We consider the usual case of externally controlled temperature $T$ and pressure $p$. At the melting temperature of the pure $A$ crystalline phase in the $A B$ mixture, the chemical potential of the $A$ particles in the crystal equals the chemical potential of the $A$ particles of the liquid mixture. The chemical potential is the Gibbs free energy per particle. The change in Gibbs free energy per $A$ particle at melting, $\Delta G_{\text {trans }, A}$ can be divided into two terms, $\Delta G_{\text {fus }, A}$ and $\Delta G_{\text {mix }, A}$, where $\Delta G_{\text {fus }, A}$ is the change in Gibbs free energy per $A$ particle upon melting an $A$ crystal into pure $A$ liquid, and $\Delta G_{\text {mix }, A}$ is the change in Gibbs free energy per $A$ particle going from pure to mixed liquid. The melting temperature $T_{\text {fus }, A}$ is determined by

$$
\Delta G_{\text {trans }, A}=\Delta G_{\text {fus }, A}\left(T_{\text {fus }, A}\right)+\Delta G_{\text {mix }, A}\left(T_{\text {fus }, A}\right)=0 .
$$

The case of pure $A\left(x_{B}=0\right)$ will be denoted by an asterisk, thus $T_{\text {fus }, A}^{*}$ is the melting point of the pure $A$ crystal into pure $A$ liquid. In this case, $\Delta G_{\text {trans }, A}=\Delta G_{\text {fus }, A}^{*}$ and since $G=H$ $-T S$, one has 


$$
\Delta H_{\text {fus }, A}^{*}-T_{\text {fus }, A}^{*} \Delta S_{\text {fus }, A}^{*}=0,
$$

where here and henceforth all thermodynamic quantities are per $A$ atom.

When deriving the standard expression for the freezing point depression, one makes the approximation that crystal and liquid have the same specific heats, i.e., that $\Delta H_{\text {fus }, A}$ and $\Delta S_{\text {fus }, A}$ are temperature independent, or from Eq. (2),

$$
\Delta H_{\text {fus }, A}-T_{\text {fus }, A}^{*} \Delta S_{\text {fus }, A}=0 .
$$

In the general case where $x_{B} \neq 0$ one has from Eq. (1),

$$
\Delta H_{\text {fus }, A}-T_{\text {fus }, A} \Delta S_{\text {fus }, A}=-\Delta G_{\text {mix }, A},
$$

and by using Eq. (3) to eliminate $\Delta S_{\text {fus }, A}$ in Eq. (4) one obtains

$$
\frac{\Delta T_{\text {fus }, A}}{T_{\text {fus }, A}^{*}}=-\frac{\Delta G_{\text {mix }, A}}{\Delta H_{\text {fus }, A}}
$$

for the melting-point depression $\Delta T_{\text {fus }, A}=T_{\text {fus }, A}^{*}-T_{\text {fus }, A}$.

In the standard textbook treatment one assumes that $\Delta H_{\text {mix }, A}=0$ and that the mixing entropy is ideal, i.e., that the mixing entropy divided by $N=N_{A}+N_{B}$ is given by $\Delta S_{\text {ideal mix }}=-k_{B}\left[x_{A} \ln \left(x_{A}\right)+x_{B} \ln \left(x_{B}\right)\right]$. This expression separates into a contribution from the $A$ particles and one from the $B$ particles. Per $A$ particle we thus have $\Delta S_{\text {ideal mix, } A}=$ $-k_{B} \ln \left(x_{A}\right)=-k_{B} \ln \left(1-x_{B}\right)$. Under these assumptions Eq. (5) for $x_{B} \rightarrow 0$ reduces to the well-known expression

$$
\frac{\Delta T_{\text {fus }, A}}{T_{\text {fus }, A}^{*}} \cong \frac{k_{B} T_{\text {fus }, A}}{\Delta H_{\text {fus }, A}^{*}} x_{B} .
$$

More generally, $\Delta H_{\mathrm{mix}, A}=0$ does not apply. In fact for the KA liquid, the mixing enthalpy is large (and negative) and this term cannot be ignored. For small concentrations of the solute, $x_{B}$, the mixing entropy is still given by $\Delta S_{\text {ideal mix }, A}$. For this more general case, Eq. (5) becomes

$$
\frac{\Delta T_{\text {fus }, A}}{T_{\text {fus }, A}^{*}}=-\frac{\Delta H_{\text {mix }, A}+k_{B} T_{\text {fus }, A} \ln \left(x_{A}\right)}{\Delta H_{\text {fus }, A}} .
$$

A negative mixing enthalpy (i.e., exotherm) clearly implies a further melting-point depression beyond that of the traditional treatments. If there is a large negative mixing enthalpy, this effect cannot be ignored.

\section{B. Crystallization}

The observed stability of a supercooled liquid mixture depends not only on its absolute (thermodynamic) stability against crystallization but also on kinetic effects. The less supercooled the liquid is the larger is the critical nucleus. This means that it takes a longer time before a critical nucleus is generated by a thermal fluctuation, i.e., the supercooled liquid is more stable. At a given temperature, the more negative $\Delta G_{\mathrm{mix}, A}$ becomes, the more stable the supercooled liquid is. To see this divide the creation of a crystal nucleus of pure $A$ particles in the mixture

$$
N_{A}(\operatorname{mix}) \rightarrow N_{A}\left(\text { crystal }, x_{A}=1\right)
$$

into two steps:

$$
\begin{aligned}
& N_{A}(\operatorname{mix}) \rightarrow N_{A}\left(\text { liquid }, x_{A}=1\right), \\
& N_{A}\left(\text { liquid }, x_{A}=1\right) \rightarrow N_{A}\left(\operatorname{crystal}, x_{A}=1\right) .
\end{aligned}
$$

The first reaction is a composition fluctuation from the mixture to a pure liquid domain of $A$ particles, the second is crystallization. The crystallization is (qualitatively) described by classical nucleation theory (CNT). In its simplest formulation, the number of particles, $N^{*}$, in the critical nucleus is given ${ }^{6}$ by

$$
N^{*}=\frac{32 \pi \gamma_{\infty}^{3}}{3 \rho_{c}^{2} \Delta \mu^{3}},
$$

where $\gamma_{\infty}$ is the solid-liquid surface tension, $\rho_{c}$ is the crystal number density, and $\Delta \mu \equiv \Delta \mu_{A}$ is the change in Gibbs free energy per $A$ particle by going from crystal to liquid. When the creation of a pure $A$ crystal takes place in a mixture, the lowering of Gibbs free energy by the crystallization process is reduced by $\delta \mu=-\Delta G_{\text {mix }, A}=-\Delta H_{\text {mix }, A}+T_{\text {fus }, A} \Delta S_{\text {ideal mix }, A} \approx$ $-\Delta_{\text {mix }, A} u_{\text {pot }}-k_{B} T_{\text {fus }, A} \ln \left(x_{A}\right)$. Since the size of the critical nucleus $N^{*}$ varies as $N^{*} \propto\left(\Delta \mu_{A}\right)^{-3}$, when $\Delta \mu_{A}$ is replaced by $\Delta \mu_{A}-\delta \mu_{A}$ to lowest order, the change in critical nucleus size $\delta N^{*}$ is given by $\delta N^{*} / N^{*}=3 \delta \mu_{A} / \Delta \mu_{A}$, thus

$$
\frac{\delta N^{*}}{N^{*}}=-3 \frac{\Delta_{\text {mix }, A} u_{\text {pot }}\left(x_{A}\right)+k_{B} T_{\text {fus }, A} \ln \left(x_{A}\right)}{\Delta \mu_{A}} .
$$

The stability criteria indicate that if a binary mixture crystallizes into, e.g., an $A B$-type crystal, a negative mixing energy will enhance the tendency of crystallization by decreasing the size of the critical nucleus. Confirming this, we find in our simulations that increasing the fraction of $B$ particles to $x_{B}=0.5$ for KA-type BLJ mixtures, results in systems that quickly crystallize into an $A B(\mathrm{CsCl}$ structure) crystal. This is consistent with the results of Fernandez and Harrowell, ${ }^{7}$ who found that the $T=0$ equilibrium phase of the KA BLJ mixture consists of coexisting pure $A(\mathrm{fcc})$ and $A B(\mathrm{CsCl}$ structure) crystals.

CNT can be used to estimate the crystallization time, $\tau$, of the solvent in a supercooled binary mixture at temperature $T<T_{\text {fus }, A}$, given that the crystallization time is known for "similar" systems that are more prone to crystallization. In the derivation below we consider crystallization in systems with different strength of solvation energy, given by the attraction between solvent and solute particles (Sec. III). by $^{8,9}$

According to CNT, the nucleation rate in CNT is given

$$
k_{\mathrm{CNT}}=Z f_{e}\left(N^{*}\right) \rho_{c} e^{-\beta \Delta G\left(N^{*}\right)},
$$

where $Z$ is the "Zeldovich" factor, $f_{e}\left(N^{*}\right)$ is the "forward rate" per particle of the growth of the critical nucleus with $N^{*}$ particles, and $\Delta G\left(N^{*}\right)$ is the Gibbs free energy barrier for the critical nucleus,

$$
\Delta G\left(N^{*}\right)=\frac{16 \pi \gamma^{3}}{3 \rho_{c} \Delta \mu^{2}} .
$$

We now consider the ratio between the nucleation rates $k_{j} / k_{i}$ of two similar systems. This ratio consists of a preexponential factor and the difference between the two free- 
energy barriers in an exponential. The later difference

$$
\Delta G_{i}\left(N^{*}\right)-\Delta G_{j}\left(N^{*}\right)=\Delta G_{i}\left(N^{*}\right)\left(1-\left(\frac{\Delta \mu_{i}}{\Delta \mu_{j}}\right)^{2}\right)
$$

(assuming that the surface tensions of the critical nuclei are the same). In the case of crystallization of the solvent from a binary mixture with particle fraction $x_{A}$ of the solvent particles, the change in free energy per $A$ particle is given by

$$
\Delta \mu=-\Delta \mu_{\mathrm{fus}}+\Delta_{\mathrm{mix}, A} u_{\mathrm{pot}}-T \Delta S_{\mathrm{mix}, A} .
$$

It is not necessary to assume ideal mixing, only that the entropy per $A$ particle is the same for the binary mixtures with different strength of solvation forces. The difference in Gibbs free energy change per particle for crystallization in the two systems only differs by the potential energy per $A$ particles in the two mixtures (with the same solute concentration)

$$
\Delta \mu_{j}=\Delta \mu_{i}-\Delta u_{i j}\left(x_{A}\right),
$$

where $\Delta u_{i j}\left(x_{A}\right) \equiv u_{A, \text { pot }, i}\left(x_{A}\right)-u_{A, \text { pot }, j}\left(x_{A}\right)$. Using the relation between $\Delta \mu_{j}$ and $\Delta \mu_{i}$ one obtains

$$
\Delta G_{i}\left(N^{*}\right)-\Delta G_{j}\left(N^{*}\right) \approx-\Delta G_{i}\left(N^{*}\right) \frac{2 u_{i j}}{\Delta \mu_{i}} .
$$

The (forward) rate at which a crystal grows $f_{e}\left(N^{*}\right)$ is proportional to the area of the crystal and to the mean speed of the particles that hits the crystal surface. The speed is proportional to the self-diffusion constant, $D$, of solvent particles in the mixture.

The self-diffusion constant for a supercooled highly viscous liquid decreases faster than an Arrhenius expression with the degree of supercooling and with increasing solvation energy. In contrast, the pre-exponential factor $Z$ and the area of the critical nucleus vary only algebraic with $\Delta \mu(T)$, so to first order

$$
\frac{Z_{j} f_{e, j}\left(N_{j}^{*}\right)}{Z_{i} f_{e, i}\left(N_{i}^{*}\right)} \approx \frac{D_{j}\left(x_{A}\right)}{D_{i}\left(x_{A}\right)},
$$

and the ratio between the nucleation rates $k_{j} / k_{i}$ or mean nucleation time $\tau_{i} / \tau_{j}$ is

$$
\frac{k_{j}}{k_{i}}=\frac{\tau_{i}}{\tau_{j}} \approx \frac{D_{j}}{D_{i}} e^{-\beta \Delta G_{i}\left(N^{*}\right)\left(2 u_{i j} / \Delta \mu_{i}\right)}
$$

from which the nucleation time for a system can be estimated from similar systems if the nucleation times $D$ and $u_{A, \text { pot }}$ are known.

We studied four MKA systems (Sec. III) to estimate the mean nucleation time of the KA system of $N_{A}=800$ and $N_{B}$ $=200$ particle at the particle density $\rho=1.2$ and temperature $T=0.40$.

\section{CRYSTALLIZATION IN BINARY MIXTURES}

\section{A. Wa, KA, and MKA BLJ mixtures}

The simplest case of a realistic mixture is that given by the semiempirical Lorentz-Berthelot (LB) rules ${ }^{10}$ relating the energy parameters $\sigma$ and $\epsilon$ in the pair potentials between the different species of the $A B$ mixture as follows:

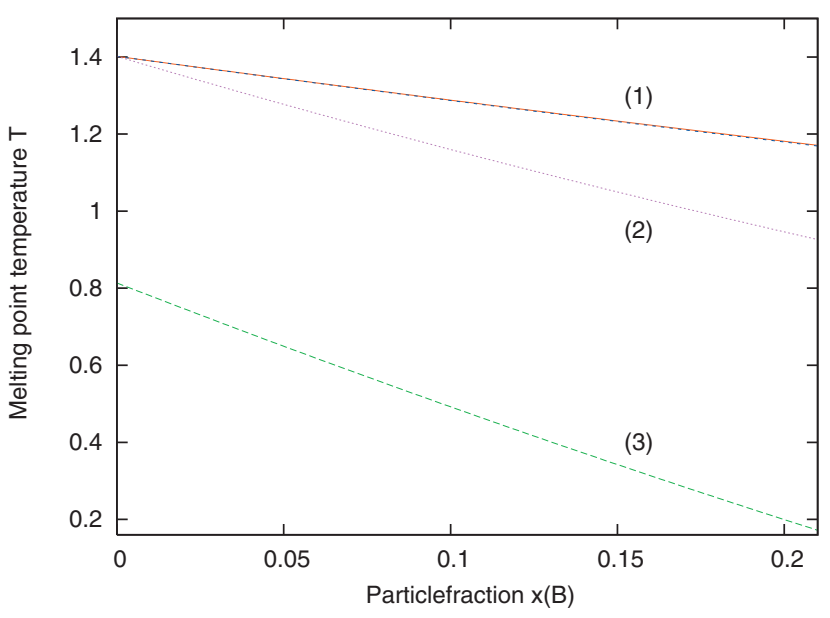

FIG. 1. (Color) Melting point temperature $T_{\text {fus }, A}$ of the solvent of $A$ particles at the external pressure $p=10$ as a function of the particle fraction $x_{B}$ of solute particles, and in the concentration interval $x_{B} \in[0,0.2]$, where the system crystallizes into a crystal of pure (fcc) $A$ particles. (1) With full line is $T_{\text {fus }, A}\left(x_{B}\right)$ for an ideal mixture and the Wa model (Ref. 1) (no visible difference), (2) with small dashes is for the MKA2 system $(n=2)$ (see Sec. III), and (3) with long dashes is the present new mixture without like-particle attractions (see Sec. IV).

$$
\begin{aligned}
& \sigma_{A B}=\left(\sigma_{A A}+\sigma_{B B}\right) / 2 \\
& \epsilon_{A B}=\sqrt{\epsilon_{A A} \epsilon_{B B}} .
\end{aligned}
$$

These rules apply for atoms with weak dispersion attractions, ${ }^{11}$ and they work well for simple mixtures of, for instance, noble-gas atoms, ${ }^{10}$ i.e., for interactions between spherically symmetric atoms with unperturbed valenceelectron orbitals.

There are two standard models for highly viscous supercooled binary mixtures, the model introduced by Wa (Ref. 1) and the model by KA. ${ }^{2}$ Both models involve LJ,

$$
u_{\mathrm{LJ}}\left(r_{i j}\right)=4 \epsilon_{i j}\left[\left(\frac{\sigma_{i j}}{r_{i j}}\right)^{12}-\left(\frac{\sigma_{i j}}{r_{i j}}\right)^{6}\right],
$$

between particle $i$ and $j$. The Wa model obeys the LB rules by having

$$
\sigma_{A B}=\left(\sigma_{A A}+\sigma_{B B}\right) / 2,
$$

with $\sigma_{B B}=1 / 1.2 \sigma_{A A}$ and $\epsilon_{A B}=\epsilon_{A A}=\epsilon_{B B}=1$. The KA model strongly disobeys the LB rules. The LJ potential parameters for the KA mixture are $\sigma_{A B}=0.8 \sigma_{A A}, \sigma_{B B}=0.88 \sigma_{A A}, \epsilon_{A B}$ $=1.5 \epsilon_{A A}$, and $\epsilon_{B B}=0.5 \epsilon_{A A}$. The fact that the binding energy of the small solute particle to a solvent particle is three times larger than the binding energy between two solute particles results in a tendency for the small and mobile solute $B$ particles to "glue" to the $A$ particles. The KA mixture has a significant nonideal mixing energy and it is very stable against crystallization, much more so the Wa mixture. So far the KA system has not been crystallized.

We estimated ${ }^{12}$ the melting-point temperature at constant pressure $\left(p \sigma_{A A}^{3} / \epsilon_{A A}=10\right)$ for small solute concentrations $x_{B}$ using Eq. (7) for the different models. The result is shown in Fig. 1. The melting point depression by mixing is traditionally given at constant pressure. Most molecular dynamics 


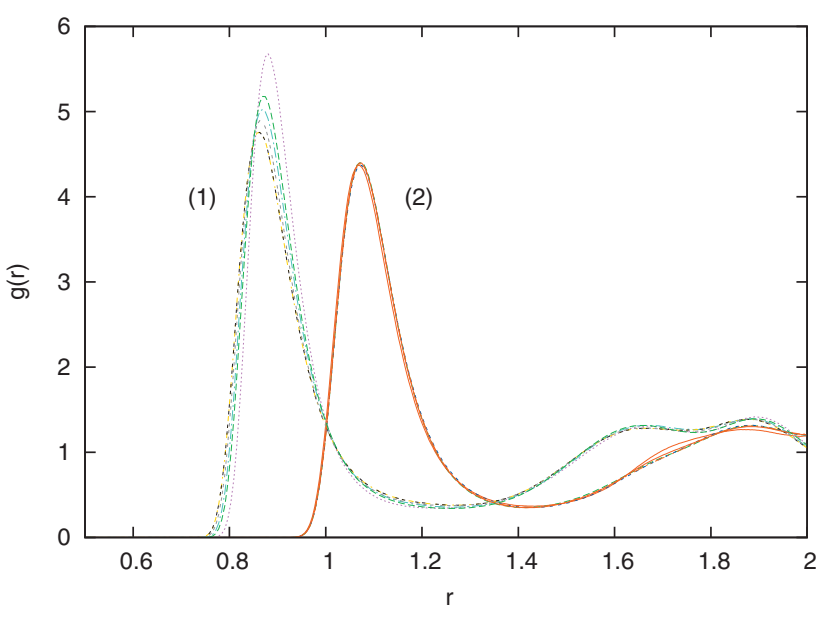

FIG. 2. (Color) Radial distribution functions for the solvent-solute particles, $g_{A B}(r)$ and the solvent-solvent particles $g_{A A}(r)$ at $T=0.40$ and $\rho=1.2$, and for the four systems with data given in Table I. (1) is $g_{A B}(r)$ and (2) is $g_{A A}(r)$.

(MD) simulations are, however, performed for isochores where $T_{\text {fus, } A}\left(x_{B}\right)$ depends on the partial volumes of the species in the mixture.

As can be seen from the figure, the negative mixing energy lowers the melting point temperature significantly. The Wa model is indeed more prone to crystallization than the other models and recently, Pedersen et al. ${ }^{5}$ reported crystallization of the Wa model after lengthy computer runs. The crystal is the $\mathrm{MgZn}_{2}$ phase consisting of particles in the ratio 1:2, different from the 1:1 ratio defining the Wa BLJ. Thus concentration fluctuations precede (or at least correlate with) crystal formation.

The KA model has served as a standard computer model for investigations of long time behavior of highly viscous liquids. With the continuous growth of computer capability, it is now realistic to simulate viscous system over times which approach microseconds. It is therefore useful to know whether this system will crystallize within this time interval. According to the previous section, one can estimate the nucleation time $\tau(\mathrm{KA})$ in the KA system from the nucleation times $\tau$ (MKA) in similar systems. The similar systems must be such that the distribution of the solvent particles is the same but with a weaker mixing energy, given by the mean potential energy of the solvent particles in the mixtures. We performed four sets of simulations with a scaled energy of attraction of the solute particle $B$ given by the energy parameter

$$
\epsilon_{A B}(\mathrm{MKA})=\epsilon_{A B}\left(\sigma_{A B} / \sigma_{A A}\right)^{n},
$$

$$
\epsilon_{B B}(\mathrm{MKA})=\epsilon_{B B}\left(\sigma_{B B} / \sigma_{A A}\right)^{n},
$$

with $n=1.5,2,2.5$, and 3 . In the following, the four systems are labeled MKA1.5, MKA2, MKA2.5, and MKA3, respectively. The scaled energies of the solute and its interaction with the solvent particles do not affect the distribution of the solvent particles. Figure 2 shows the radial distribution functions, $g_{A, A}(r)$ and $g_{A, B}(r)$ for the distribution of $A-A$ interactions and $A-B$ interactions. Whereas $g_{A, A}(r)$ is only little affected by the weaker binding to the solvent particles, $g_{A, B}(r)$ shows a reduced probability for a direct attachment of the $B$ particles. The corresponding mean potential energy of the solvent particles in the mixture at $\rho=1.2$ and $T=0.40$ are shown in Table I. The reduced energy of the solute particles leads to a corresponding reduction in the (negative) potential energy, $u_{A, \text { pot }}(x)$.

\section{B. Crystallization of a MKA binary mixture}

In order to investigate the crystallization properties of the KA and MKA mixtures, we performed MD simulations of 1000 (occasionally 10000 ) particles of the MKA version in both the $N V E$ and the $N V T$ ensembles. The idea is that the KA system crystallizes by a mechanism similar to that of the modified system.

The standard time-reversible leap-frog (NVT) algorithm ${ }^{13}$ was used. In the following, data are given in unit length $\sigma_{A A}$, unit energy $\epsilon_{A B}$, and unit time $\sigma_{A A} \sqrt{m / \epsilon_{A A}}$ $\left(\approx 2 . \times 10^{-12} \mathrm{~s}\right.$ in argon units). The software used has been described elsewhere. ${ }^{14}$ It utilizes a double sorting of neighbor particles that makes it possible to simulate $1 \mu$ s within 2-3 days of computing on a standard computer.

With the MKA2 system, we did not detect crystallization for temperatures above 0.45 . In the temperature interval $[0.39,0.45]$, a drop in pressure taking place typically after $\approx 10^{6}$ time units indicates that the system phase separates such that the $A$ particles form fairly large regions with no $B$ particles present. Linked to this phase separation is a crystallization of the $A$ particles. Ten simulations with the MKA2 system were performed in the $[0.39,0.45]$ temperature interval. After $2 \times 10^{6}$ time steps $(\approx 4 \mu \mathrm{s})$ eight of these ten simulations phase separated with crystallization of the $A$ particles. Figure 3 shows a representative example of the crystallization, giving the positions of the particles after crystallization at $T=0.40$ (NVT MD). There is a large region of pure $A$ particles showing clear crystalline order.

TABLE I. KA and MKA systems at $T=0.40$ and $\rho=1.2$. Length unit: $\sigma_{A A}$, time unit: $\sigma_{A A} \sqrt{m / \epsilon_{A A}}$ $\left(\approx 2 . \times 10^{-12} \mathrm{~s}\right.$ in argon units $)$.

\begin{tabular}{llcccc}
\hline \hline System & $n$ & \multicolumn{1}{c}{$D_{A}$} & $u_{\text {pot }, A}$ & $\tau$ & $\ln (\tau D)$ \\
\hline MKA3 & 3 & $4.54 \times 10^{-5}$ & -5.582 & $2.70 \times 10^{5}$ & 2.51 \\
MKA2.5 & 2.5 & $3.64 \times 10^{-5}$ & -5.696 & $4.92 \times 10^{5}$ & 2.89 \\
MKA2 & 2 & $1.79 \times 10^{-5}$ & -5.850 & $1.82 \times 10^{6}$ & 3.48 \\
MKA1.5 & 1.5 & $9.99 \times 10^{-6}$ & -6.043 & $2.97 \times 10^{6}$ & 3.46 \\
KA & 0 & $9.18 \times 10^{-7}$ & -6.584 & $\approx 5 . \times 10^{7}$ & $(3.83)$ \\
& & & (extrapolated) & \\
\hline
\end{tabular}




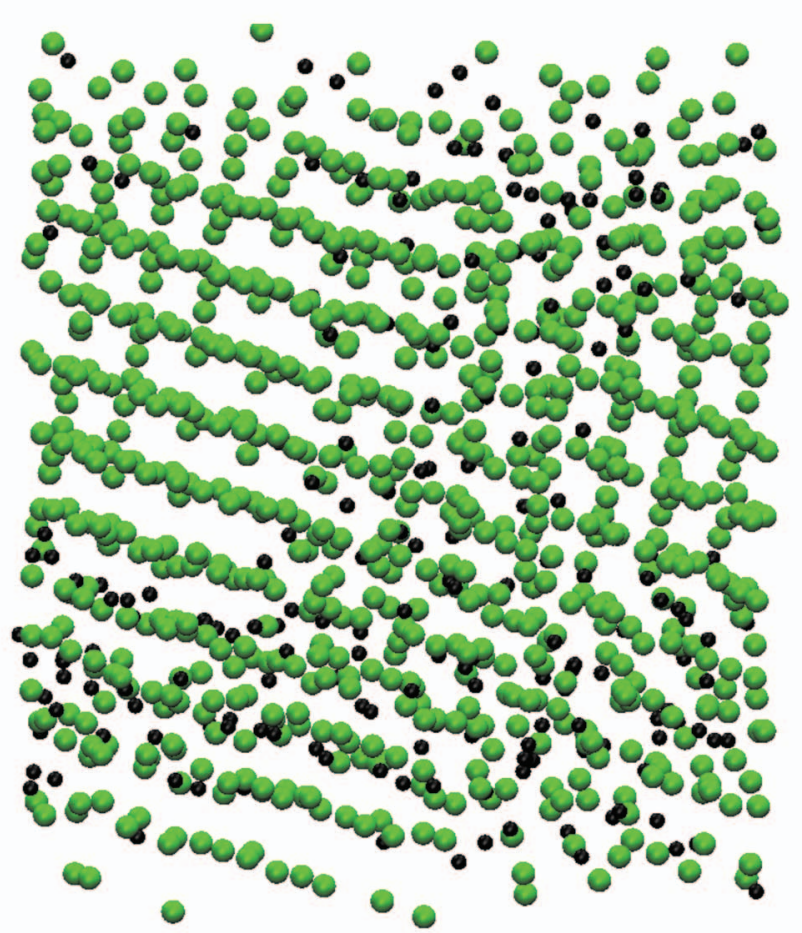

(a)

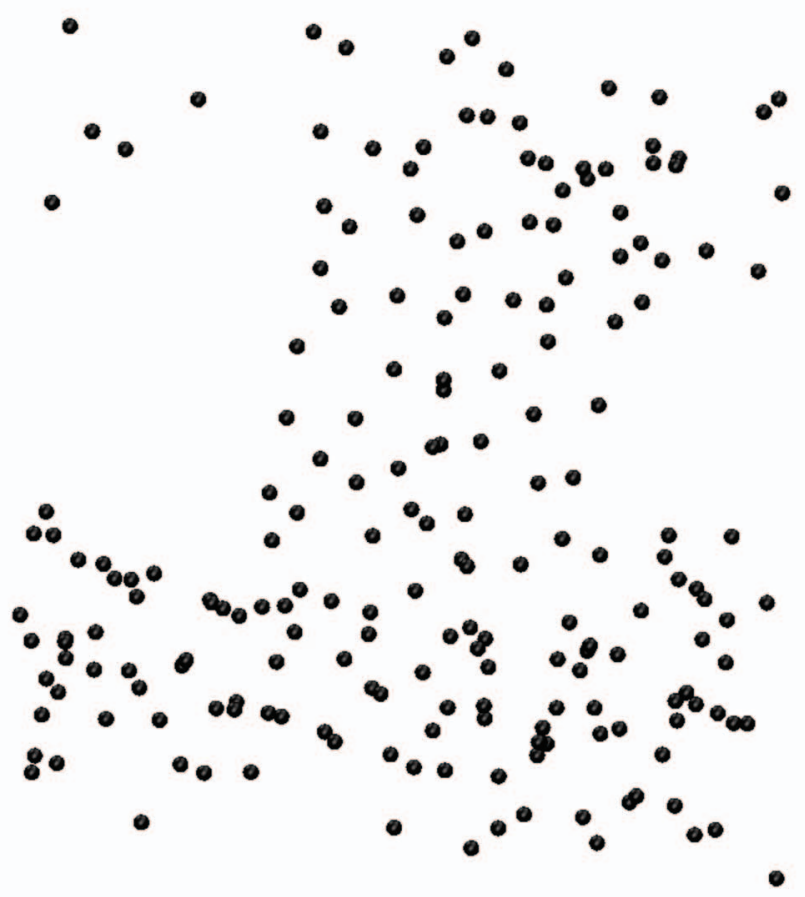

(b)

FIG. 3. (Color) (a) Particle positions projected onto a plane for the MKA2 liquid [the large $(A)$ particles are green, the small $(B)$ particles are black]. The system is shown after $1.5 \mu \mathrm{s}$ of simulation (argon units) at $T=0.40$ and density 1.2 (dimensionless units). After this simulation time, the $A$ particles phase separated and formed a large crystal, as is clear in the second figure showing the same configuration with all $A$ particles removed.

\section{Estimation of the crystallization time for the KA binary mixture}

According to the theory in Section II B, the nucleation time in a KA mixture can be estimated from the nucleation time in similar systems. We performed MD simulations for four systems with different scaled solute-solute and solutesolvent energy, given by the power $n=1.5,2,2.5$, and 3 , respectively [see Eqs. (24) and (25)]. The mean nucleation times, $\tau$, obtained from a maximum likelihood estimate ${ }^{15}$ based on twelve independent simulations for each of the four MKA mixtures at $T=0.40, \rho=1.2$, and $x_{A}=0.8$ are shown in Table I, together with the corresponding values of the selfdiffusion constants $D_{A}$, and the mean potential energies $u_{A \text {,pot}}$, per $A$ particles in the binary mixtures.

In principle the estimation of nucleation times can be performed in two different ways (1) by linear extrapolation of $\ln \tau(n)$ as a function of $n$ and (2) from $\ln \left[\tau_{i} D_{i}\left(u_{i}\right)\right]$, according to Eq. (19), as a function of the mean potential energy of the nucleating solvent particles in the binary mixture. The data in Table I is used for the estimations of the crystallization time for the KA system. Method 1 predicts a mean nucleation time for the classical KA mixture of the order 5 $\times 10^{7}$ time units (0.1 $\mu$ s argon units). Concerning method 2 , we notice that although the uncertainty in the nucleation time $\tau_{i}$ is large, the variation in $\ln \left[\tau_{i} D_{i}\left(u_{i}\right)\right]$ is relatively small and in the interval $[2.5,3.5]$ for the four MKA systems. This indicates that the main effect of the resistance against crystallization in the systems arises indirectly from the increased viscosity by increasing the solvation energy, not directly from the solvation energy itself, given by the term $u_{i}$ in Eq. (19). Using method 2 and with $\ln \left[\tau_{i} D_{i}\left(u_{i}\right)\right] \approx 3.83$ gives a nucleation time of $5 \times 10^{7}$.

Our conclusion, based on the two methods, is that the KA mixture will nucleate roughly within $5 \times 10^{7}$ time units, a time interval which is realistic for computer simulations in the near future.

\section{A NEW BINARY MODEL SYSTEM}

With the general theory for melting point depression and nucleation in mind it is possible to change existing binary models in such a way that they are faster to simulate and even less prone to crystallization. The idea is to construct a new mixture with only repulsive forces between $A-A$ and $B-B$ particles, but where the attraction between $A$ and $B$ particles that is crucial for preventing crystallization is maintained. With LJ potentials between particles $i$ and $j$, the modified potentials are

$$
u_{i j}\left(r_{i j}\right)= \begin{cases}u_{\mathrm{LJ}}\left(r_{i j}\right)-u_{\mathrm{LJ}}\left(r_{i j}(\mathrm{cut})\right), & r_{i j}<r_{i j}(\mathrm{cut}), \\ 0, & r \geq r_{i j}(\mathrm{cut}),\end{cases}
$$

with

$$
\begin{aligned}
& r_{i, i}(\text { cut })=2^{1 / 6} \sigma_{i i}, \\
& r_{A B}(\text { cut })=2.5 \sigma_{A B} .
\end{aligned}
$$

A priori, it is to be expected ${ }^{16}$ that the structure of the mixture only deviates marginally from the corresponding KA or MKA models detailed above because the repulsive parts of the potential interactions are not changed. The new binary model is more stable, and we have so far not been able to crystallize it (for a particle fraction $x_{B}=0.2$ ). The new system(s) is three times faster to simulate than the corresponding KA or MKA mixtures. 


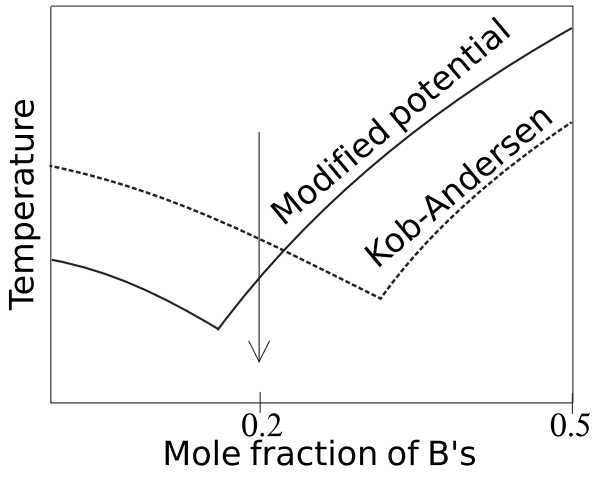

FIG. 4. Schematic illustration of the change in melting point temperature, $T_{m}\left(x_{B}\right)$ as a function of the particle fraction, $x_{B}$ by removing the attraction between the $A, A$ and $B, B$ pair interactions.

The modification can be applied to any (MKA and KA) of the BLJ mixtures investigated above. We used it for the MKA2 system with $n=2$. The new system was cooled and equilibrated at a new and lower temperature until the diffusion constant remained constant. The system was then simulated $5 \times 10^{7}$ time units (corresponding to $\approx 0.1 \mu s$ ) for nine temperatures in the interval $T \in[0.25,0.40]$. There are two reasons for the resistance against crystallization by supercooling. First, the melting point temperature, $T_{\text {fus }, A}^{*}$, of the pure solvent particles is lowered significantly (see Fig. 1). Second, the relative importance of the mixing energy, $\Delta_{A, \text { mix }} u_{\mathrm{pot}}\left(x_{B}\right)$ is bigger due to a stronger violation of the LB rules for the energy and thereby a lower melting point temperature and an increase in the size of the critical fcc nucleus.

The question arises, however, whether the new system is supercooled or simply a highly viscous equilibrium liquid. As mentioned most MD simulations are performed at constant density. Thus, the result shown in Fig. 1 for $p=10$ cannot be used to obtain the melting point temperature in the new system at $\rho=1.2$ and $x_{B}=0.2$, and to determine whether the system is in a supercooled state. The MKA2 mixture crystallizes into a pure $A$ particle fcc structure for small particle fraction $x_{B}$, and into a $\mathrm{CsCl}(A, B)$ crystal for $x_{B} \approx 0.5$. In between, there might be other stable crystal arrangements. ${ }^{17}$ The relative strength of the binding energy between $A$ and $B$ particles increases when the attraction between $A, A$ and $B, B$ particles is removed, but with unchanged attraction between $A$ and $B$ particles. This change stabilizes the CsCl-like crystal relative to the liquid and the melting point temperature at $x_{B}=0.5$ increases, whereas the melting point temperature of the fcc crystal for small $x_{B}$ is decreased (as shown in Fig. 1). The qualitative change in melting point temperature by the modification at constant pressure is shown in Fig. 4.

There is a simple computer experiment to investigate whether the new system at $\rho=1.2$ and $x_{B}=0.2$ is in a supercooled state at the low temperature. First we crystallized the new system at particle fraction $x_{B}=0.5$. Then the system with $\rho=1.2$ and $x_{B}=0.2$ was grafted with a $\mathrm{CsCl}$ crystal of $200 \mathrm{~A}$ and $200 B$ particles, taken from the $x_{B}=0.5$ system and surrounded by $600 \mathrm{~A}$ particles at a density $\rho=1.2$. The melting point temperature of this crystal was directly determined by heating/cooling. The melting point temperature of the $\mathrm{CsCl}$ grafted system at $x_{B}=0.2$ and $\rho=1.2$ is $T_{m} \approx 0.32$, and the new system is indeed a supercooled highly viscous liquid at the low temperatures investigated. For $T=0.30$ and below the system with the $A B$ crystal of 400 particles remained but the remaining $A$ particle crystallized into a fcc structure. The configuration is shown in Fig. 5. The computer experiment demonstrates that the supercooled system below is what corresponds to the eutectic temperature in a constant pressure system. The actual phase diagram, however, might be even more complicated than given here. ${ }^{17}$

A further advantage of the new system is that it is much faster to simulate by using a two-step sorting of nearest neighbor. ${ }^{14}$ In addition to that one can use a larger value of the time increment in the MD simulation due to the lower temperature, so not only does the mixture not crystallize but it can also be cooled further down under equilibrium condition (see Figs. 7-9) and followed for longer times.

The structure of the new mixture is, as expected, almost the same as in the KA and the modified KA mixtures. Figure 6 show the radial distribution functions, $g_{\alpha, \beta}(r)$ for the new mixture and for the corresponding MKA2 mixture. The similarity is well known. The so-called WCA system ${ }^{16}$ with only repulsive LJ forces has been used in perturbation theories for dense liquid. As can be seen from the figure, only the distribution, $g_{B, B}(r)$ of the $B$ particles is affected.

The MKA2 and the new mixture were cooled down from $T=1.5$. The MKA mixtures crystallize as mentioned in the temperature interval $T \in[0.39,0.45]$, but it is possible to quench the MKA2 down to $T=0.375$ and still determine the self-diffusion constant $D$. The new mixture was cooled down to $T=0.25$. At each (low) temperature, the system was followed $5 \times 10^{7}$ time units $(\approx 0.1 \mu \mathrm{s})$. The diffusion constant at $T=0.25$ for the $A$ particles is $D_{A} \approx 1.0 \times 10^{-8}$. The $B$ particles behave in a similar way, but as the temperature is decreased, the ratio $D_{B} / D_{A}$ changes from 1.7 for $T=1.5$ to 9.7 for $T=0.25$. The values of the self-diffusion constants $D(T)$ were obtained from the slopes of the mean-square displacements as function of time. At low temperatures, the ballistic and diffusive time regimes are very well separated. A log-log plot of the mean-square displacement for different temperatures shows this separation (Fig. 7).

The diffusion constants for $A$ and $B$ particles are shown in Fig. 8 together with low temperature data for the MKA2 mixture ( $A$ particles) and the KA mixture. It is possible to scale the diffusion constants for the three mixtures as is demonstrated in Fig. 9, where $\log (D)$ for the MKA2 mixture and the KA mixture are plotted as a function of $1 /(T * 0.806)$ (MKA2) and $1 /(T * 0.714)(\mathrm{KA})$. A similar "fragility invariance" for a system with different repulsive power potentials $\left(r^{-n}\right)$ was reported in Ref. 18.

\section{DISCUSSION}

The highly viscous fluid state below the equilibrium melting point is found in many systems ranging from simple organic single-component systems and alloys to complex biochemical substances and ionic liquids. Along with the increasing interest in this state of matter it is important to have models that are not prone to crystallization. Based on the 


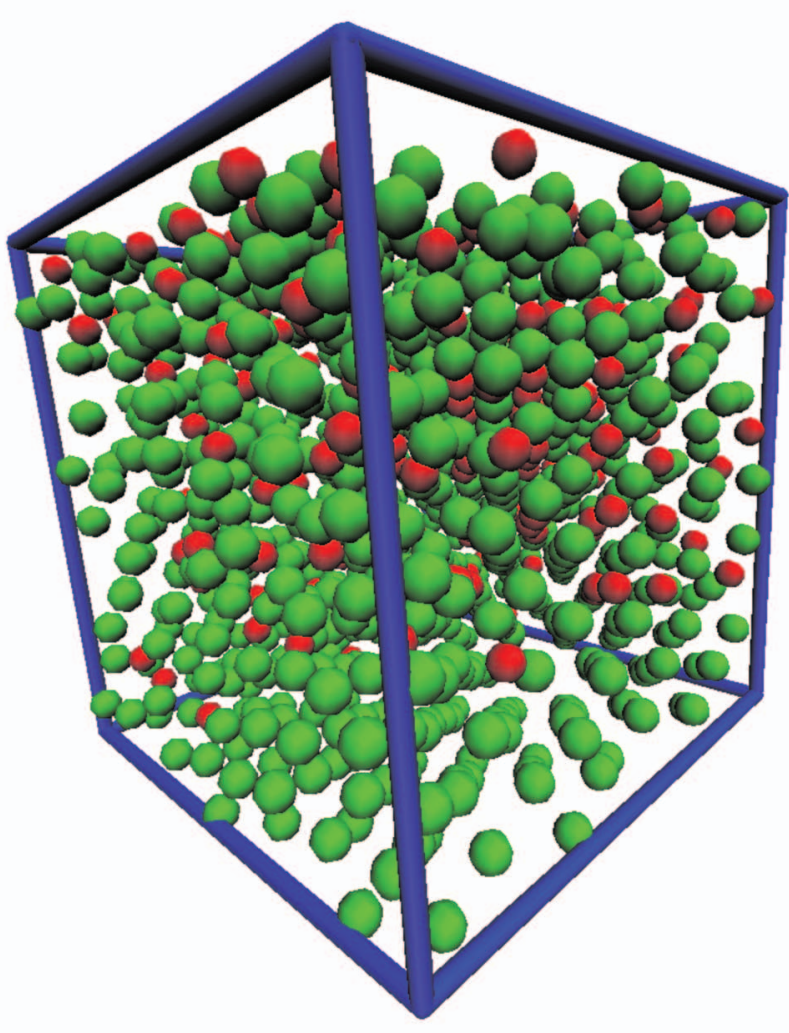

(a)

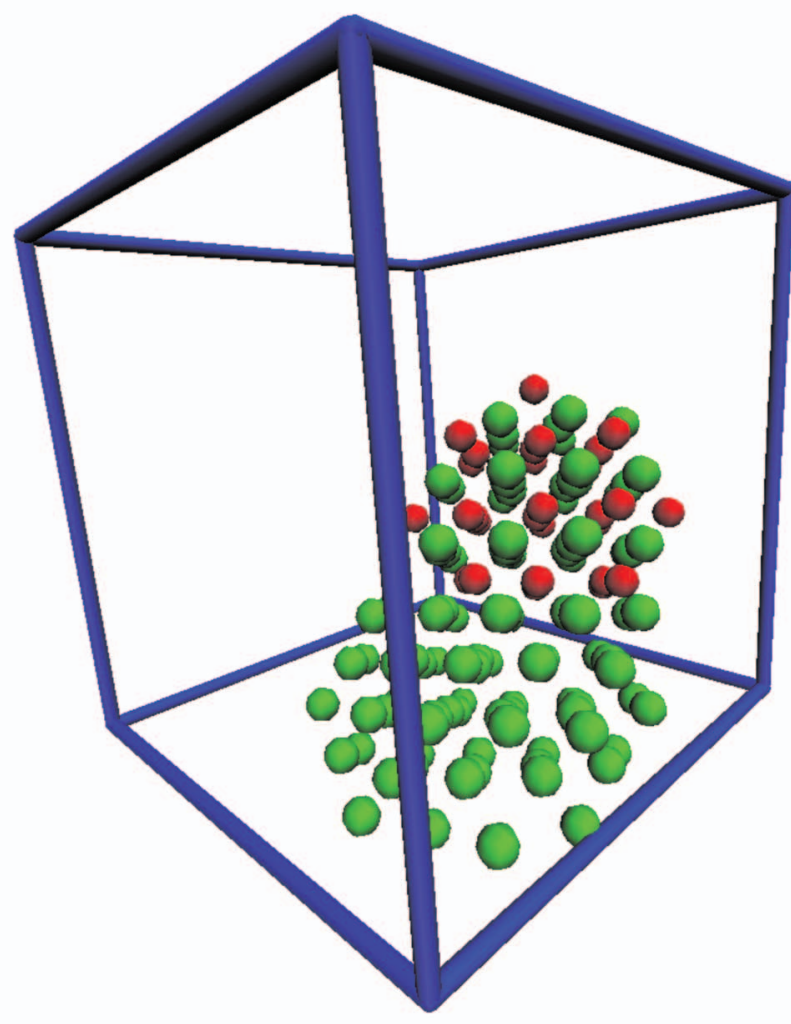

(b)

FIG. 5. (Color) Particle positions of the frozen system (Sec. IV) after they first were grafted with a small $\mathrm{CsCl}(A, B)$ crystal and at $T=0.30$. The $A$ particles are green and the $B$ particles are red. (a) The hole system of 1000 particles at the density $\rho=1.2$ and $x_{B}=0.20$. (b) A central part of the frozen system where the two coexisting crystal forms, fcc $(A)$ and $\mathrm{CsCl}(A B)$ clearly can be seen. (The frozen system contains several defects.)

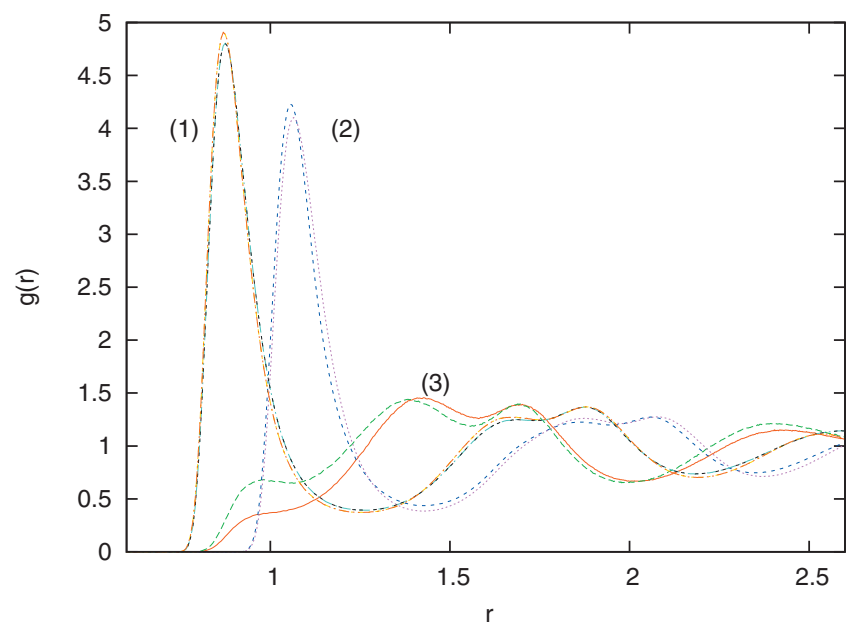

FIG. 6. (Color) Radial distribution functions (1) $g_{A B}(r),(2) g_{A A}(r)$, and (3) $g_{B B}(r)$, for the distribution of particles at the density $\rho=1.2$ and the temperature $T=0.50$ for a mixtures with particle fraction $x_{A}=0.8$ of $B$ particles. Full line is for the MKA2 mixture, the dashed curve is for the new mixture.

CNT in Sec. II and the simulations of the modified systems in Sec. III we estimate that the crystallization time for the standard KA mixture at $T=0.40$ is of the order $5 \times 10^{7}$ time units $(\approx 0.1 \mu$ s argon units $)$.

The general thermodynamic theory for (equilibrium) melting and CNT show that an exothermic mixing suppresses crystallization. On the other hand, it is the exponential decreasing self-diffusion near the glass transition in highly viscous liquid that is the dominating factor for the stabilization of highly viscous liquid (Table I). Theory also gives an indication of how to create models that suppress crystallization by cooling. We have given one simple example by starting from the modified the KA model but it is straightforward to extend the model to include other interactions and more refined energy functions without loosing stability against crystallization. A general recipe of energy functions for stable highly viscous liquids is, however, difficult to set up, since many mixtures exhibit "reacting systems" with crystals with mixed compositions, where an increased bind-

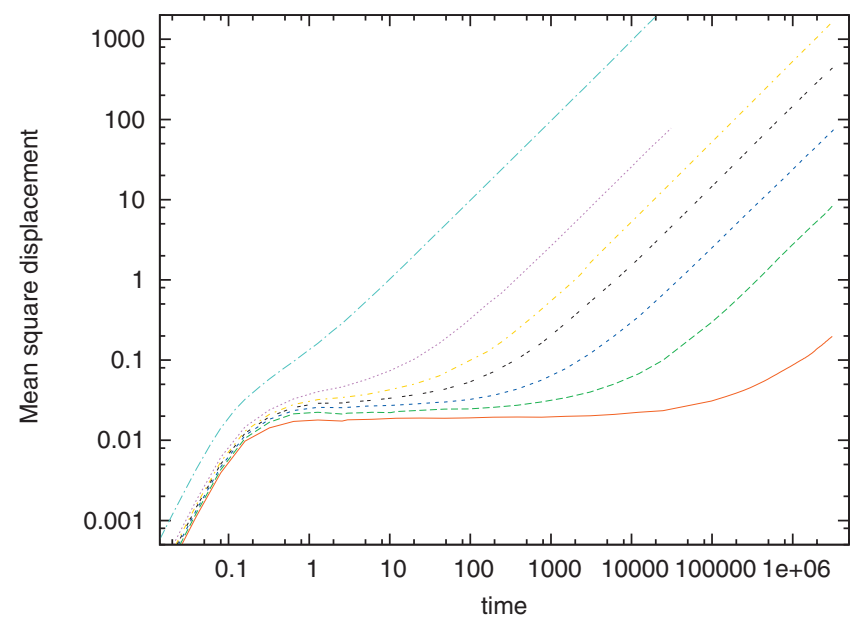

FIG. 7. (Color) log-log plot of the mean-square displacement for the $A$ particles of the new BLJ as a function of time (in unit $\sigma_{A A} \sqrt{m / \epsilon_{A A}} \approx 2$. $\times 10^{-12} \mathrm{~s}$ ) and for different temperatures (from the left): $T=1.00,0.40$, $0.35,0.325,0.30,0.275$, and 0.25 , respectively. 


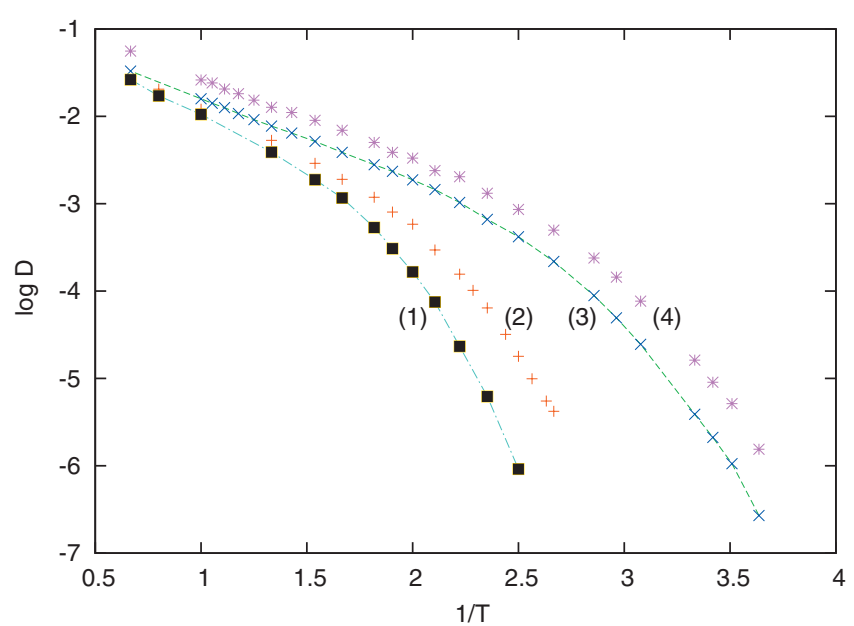

FIG. 8. (Color) An Arrhenius plot, $\log D(1 / T)$, of the self-diffusion constant $D$. (1) With filled squares and connected with dash-dots is for the KA mixture. (2) With + is $D(A)$ for the MKS2 mixture, and (3) the points given by $\times$ and connected with dashes is $D(A)$ for the new binary mixture (see Sec. III B). (4) The diffusion constants, $D(B)$ for the smaller $B$ particles in the new binary mixture are shown with $\star$.

ing or mixing energy can enhance crystallization of, e.g., $A, B$ crystals. Both the KA and the Wa models are examples of this phenomenon.

The new model enhances the relative attraction between unlike species by removing the attraction for the $A, A$ and $B, B$ interactions. Supercooled liquid (WCA) mixtures with solely repulsive LJ potentials have been proposed before, ${ }^{19,20}$ but we find that both systems studied in Refs. 19 and 20 phase separate and crystallize by cooling. By maintaining the attraction between unlike species, however, the highly viscous state is stabilized. The new system is faster to simulate than the original KA BLJ mixture. The new model has the same energy scaling parameter, $\epsilon_{\alpha \beta}$, but a much stronger

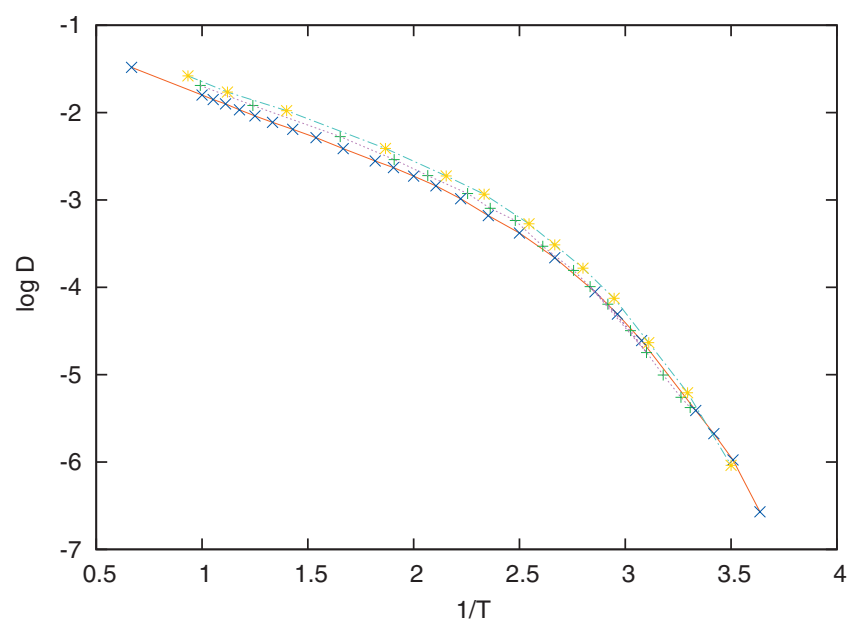

FIG. 9. (Color) With full line and $\times$ is the $\log D(1 / T)$ for the new mixture from Fig. 8. With dashes are the corresponding scaled MKA2 data $\log (D)(1 / 0.806 T)$, and with dot-dashes are the corresponding KAI data $\log (D)(1 / 0.714 T)$. violation of the energy rule in the interaction interval $r_{\alpha \beta}$ $\in\left[2^{1 / 6} \sigma_{i, i}, \infty\right]$ where the WCA potentials are zero. The result of this "cut and shift" is that the $B$ particles are almost "covalently" bounded to the $A$ particles. Thus, violation of the LB rule gives a recipe for creating fragile and stable supercooled liquid mixtures.

Note added in proof. Using high-end graphics cards (http://www.nvidia.com/cuda) we recently performed 7 independent simulations of the KA system with $T=0.40$. During the runs of $7.4 \times 10^{9}$ time steps $\left(3.7 \times 10^{7}\right)$ time units, two out of the seven samples crystallized. The estimated (mean) nucleation time $\tau$ is $1.1 \times 10^{8}$. This nucleation data are consistent with the extrapolation presented in Table I.

\section{ACKNOWLEDGMENTS}

The center for viscous liquid dynamics "Glass and Time" is sponsored by the Danish National Research Foundation's (DNRF).

${ }^{1}$ G. Wahnström, Phys. Rev. A 44, 3752 (1991).

${ }^{2}$ W. Kob and H. C. Andersen, Phys. Rev. E 51, 4626 (1995); Phys. Rev. E 52, 4134 (1995).

${ }^{3}$ T. A. Weber and F. H. Stillinger, Phys. Rev. B 31, 1954 (1985).

${ }^{4}$ U. R. Pedersen, N. P. Bailey, J. C. Dyre, and T. B. Schrøder, e-print arXiv:0706.0813; S. Toxvaerd, T. B. Schrøder, and J. C. Dyre, e-print arXiv:0712.0377.

${ }^{5}$ U. R. Pedersen, P. Harrowell, T. B. Schrøder, and J. C. Dyre (unpublsihed).

${ }^{6}$ See, e.g., A. Laaksonen, R. McGraw, and H. Vehkamäki, J. Chem. Phys. 111, 2019 (1999) and references therein.

${ }^{7}$ J. R. Fernandez and P. Harrowell, Phys. Rev. E 67, 011403 (2003).

${ }^{8}$ D. W. Oxtoby, J. Phys.: Condens. Matter 4, 7627 (1992).

${ }^{9}$ P. R. ten Wolde, M. J. Ruiz-Montero, and D. Frenkel, J. Chem. Phys. 110, 1591 (1999).

${ }^{10}$ J. S. Rowlinson, Liquid and Liquid Mixtures (Butterworths, London, 1969).

${ }^{11}$ J. O. Hirschfelder, C. F. Curtiss, and R. B. Bird, Molecular Theory of Gases and Liquids (Wiley, New York, 1954), Chap. 13.3.

${ }^{12}$ The $T_{\text {fus }, A}$ can be obtained from Eq. (7) for small $x_{B}$. The melting temperature for a pure LJ system at $p=10$ is, $T_{\text {fus }}^{*}=1.4017(3)$ and the corresponding densities are $\rho(s)^{*}=1.0630(8)$ and $\rho(l)^{*}=0.98665(3)$ of solid(s) fcc LJ particles and liquid (1). E. A. Mastny and J. J. de Pablo, J. Chem. Phys. 127, 104504 (2007), from where also the melting enthalpy, $\Delta_{\text {fus }} H^{*}$ can obtained using the (exact) Clapeyron equation, it is $\Delta_{\mathrm{fus}} H^{*}=1.675$. The mixing potential energy per solvent $A$ particle, $\Delta_{\text {mix }} u_{\text {pot }}\left(T_{\text {fus }, A}^{*}, x_{B}\right)$ at $p=10$ are obtained from MD simulations. The mixing potential energy is self-consistent with the approximation in Eq. (3) assumed to be temperature independent for small $x_{B}$.

${ }^{13}$ S. Toxvaerd, Mol. Phys. 72, 159 (1991).

${ }^{14}$ J. J. Morales, L. F. Rull, and S. Toxvaerd, Comput. Phys. Commun. 56, 129 (1989).

${ }^{15}$ S. Kay, Fundamentals of Statistical Signal Processing Volume I: Estimation Theory (Prentice-Hall, Englewood Cliffs, 1993).

${ }^{16}$ J. Weeks, D. Chandler, and H. C. Andersen, J. Chem. Phys. 54, 5237 (1971).

${ }^{17}$ F. Calvo, T. V. Bogdan, V. K. de Souza, and D. J. Wales, J. Chem. Phys. 127, 044508 (2007).

${ }^{18}$ C. De Michele, F. Sciortino, and A. Coniglio, J. Phys.: Condens. Matter 16, L489 (2004).

${ }^{19}$ S. R. Williams and D. J. Evans, Phys. Rev. Lett. 96, 015701 (2006).

${ }^{20}$ L. O. Hedges, L. Maibaum, D. Chandler, and J. P. Garrahan, J. Chem. Phys. 127, 211101 (2007). 